\title{
Análisis de las campañas contra el castigo físico a menores en España. Contenido y propuesta de estrategias para el cambio de actitudes ${ }^{1}$
}

\section{Analysis of awareness campaigns against the physical punishment of minors in Spain. Content and proposed strategies to change attitudes}

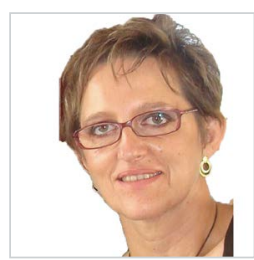

Ana Rosser Limiñana. Doctora en Psicología y Máster en Psicología de la Salud. Desde 1999 es profesora del Departamento de Comunicación y Psicología social de la Universidad de Alicante donde dirige el grupo de investigación IPSIFAM (Intervención psicosocial con familias y menores) y la Cátedra de inclusión. Sus principales líneas de investigación son la protección a la infancia en riesgo social, la prevención e intervención con víctimas de la violencia de género, y la integración universitaria de personas con discapacidad. Su última publicación, en 2018 ha sido Problemas de conducta infantil y competencias parentales en madres en contextos de violencia de género, en Gaceta Sanitaria.

Universidad de Alicante, España

ana.rosser@ua.es

ORCID: 0000-0001-9388-6696

Recibido: 11/09/2017 - Aceptado: 18/12/2017

Received: 11/09/2017 - Accepted: 18/12/2017

\section{Resumen:}

El castigo físico a los menores ha sido una práctica habitual de disciplina parental durante siglos. En los últimos tiempos se han desarrollado diferentes estrategias para su erradicación. Entre ellas, la de sensibilizar a la población sobre sus riesgos, proponiendo formas alternativas de disciplina. En este trabajo se analizan el contexto y los elementos persuasivos utilizados en las campañas publicitarias emitidas a nivel institucional en España para este fin y su efectividad. Se concluye sobre la necesidad de potenciar el desarrollo de campañas de este tipo a través de los medios de comunicación, dándoles una mayor difusión que permita mejorar su efectividad, y ofreciendo estrategias educativas alternativas al castigo físico para que se conviertan en un verdadero instrumento de cambio de actitudes.

\section{Palabras clave:}

Castigo físico a menores; Campañas publicitarias; Sensibilización; Cambio de actitudes, Análisis de contenido.

\section{Abstract:}

The physical punishment of minors has been a common practice in parental discipline for centuries. In recent times, different strategies have been developed for its eradication. Among them, raising awareness among the population about the risks, and proposing alternative forms of discipline. In this paper, we analyse the context and the persuasive features used in awareness campaigns issued at the institutional level in Spain for this purpose and its effectiveness. We have concluded that there is a need to promote the development of campaigns of this type through the media, providing them with greater reach in order to improve their effectiveness, and offering educational strategies as an alternative to physical punishment for the purpose of making these types of strategies real instruments in changing attitudes.

\section{Keywords:}

Corporal punishment of minors; Publicity campaigns; Raising awareness; Attitude changes; Content analysis.

\section{Cómo citar este artículo:}

Rosser Limiñana, A. (2018). Análisis de las campañas contra el castigo físico a menores. Contenido y propuesta de estrategias para el cambio de actitudes. Doxa Comunicación, 26, 59-80.

1 Esta investigación se ha realizado a partir de datos obtenidos en el proyecto europeo "PUNISH-ER. Eradication of the use of physical punishment on children within family and institutions" (JUST/2010/DAP3/AG/1337), financiado por la Iniciativa Daphne de la Unión Europea. Sus resultados están sirviendo de base para los objetivos de un nuevo proyecto europeo, "Hands Up" - Promoting the effective elimination of corporal punishment against children (JUST/2015/RDAP/AG/CORP). Este nuevo proyecto trata de abordar las dificultades y carencias detectadas en el proyecto Daphne Punish-er. 
Análisis de las campañas contra el castigo físico a menores en España...

\section{Introducción}

La disciplina parental incluye las conductas de los padres dirigidas a evitar y corregir el comportamiento inapropiado del menor y a lograr su conformidad y obediencia (Fauchier y Straus, 2007). Cuando hablamos de castigo físico a menores nos estamos refiriendo a una determinada pauta educativa de crianza que tiene que ver con las estrategias de disciplina parental utilizadas por los padres a la hora de educar a sus hijos mediante azotes, bofetadas, cachetes, etc.

En ocasiones, estas prácticas se han relacionado con las características de las familias, de forma que, aquellos progenitores con menos recursos (bajos ingresos, menor nivel educativo), parecen ser más proclives a utilizar el castigo corporal severo. Además, aquellos que han tenido más posibilidades de ser socializados en el uso de la violencia presentaban más predisposición a la utilización del castigo físico severo con sus hijos (Dietz, 2000; Fráchette y Romano, 2015; Juby, 2009).

Los diferentes estilos educativos que los padres emplean con los hijos tienen consecuencias en la conducta de los mismos (Baumrind,1971; Musitu y García, 2004). Diferentes investigaciones han tratado de evaluar estos efectos de la utilización de unas estrategias u otras en la conducta del menor así como en su desarrollo psicosocial (Gámez-Guadix et al., 2010a; Fergusson y Lynskey, 1997; Grogan-Kaylor, 2004; Torío, Peña e Inda, 2008). En general, se ha señalado que las estrategias de carácter más aversivo (por ejemplo, castigo físico, amenazas) están ampliamente relacionadas con numerosas consecuencias negativas y, entre ellas, con un mayor riesgo de presencia de maltrato y abuso físico sobre el niño (Gershoff, 2002) o de que el menor, a su vez, azote o golpee a otros niños (Simons y Wurtele, 2010). Algunos autores lo relacionan también con una disminución en la calidad de las relaciones padre-hijo (Zolotor et al., 2011) y con el desarrollo de problemas de ajuste psicológico (Landsford et al., 2014; Scott, Lewsey, Thompson y Wilson, 2013: Smith, Springer y Barret, 2011).

En los últimos años, el interés se ha centrado en conocer las circunstancias que llevan a los padres y madres a utilizar el castigo físico con sus hijos. A menudo los padres se muestran ambivalentes hacia el uso del castigo físico y es frecuente que manifiesten actitudes negativas hacia este pero continúan utilizándolo a pesar de no creer en su efectividad (Bunting, Webb and Healy, 2010). Otras variables más dinámicas como el estrés parental (Fráchette y Romano, 2015), cuestiones étnicas o culturales (Holden, Hawk, Smith, Singh y Ashraf, 2017; Khoury-Kassabri, Attar-Schwartz y Zur, 2014) son también importantes.

De ahí la importancia de incidir sobre estas prácticas de cara a sensibilizar a la población sobre las razones que inducen a los padres y madres a utilizarlas, sus riesgos, y posibles estrategias alternativas para corregir el comportamiento de los menores.

En este sentido, la Convención sobre los Derechos del Niño (Naciones Unidas, 1989) afirma en su artículo 19 que "los Estados deben adoptar todas las medidas legislativas, administrativas, sociales y educativas para proteger al niño contra toda forma de violencia física o mental. (Hodgkin y Newell, 2002).

Igualmente, el Comité de los Derechos del Niño (2006) incide en que:

"Abordar la aceptación o tolerancia del castigo corporal generalizada en los niños y su eliminación, en la familia y en las escuelas y otras instituciones, no sólo es una obligación de los Estados partes en la Convención. Es también es una estrategia clave para reducir y prevenir toda forma de violencia en las sociedades", (Comité de los Derechos del Niño, de las Naciones Unidas, Observación general núm. 8, 2006, párrafo 11). 
Algunos trabajos señalan un descenso en el uso del castigo físico en algunos países como Canadá (Fráchette y Romano, 2015), aunque el azote o el cachete siguen siendo práctica habitual con niños pequeños (Zolotor et al., 2011). Sin ánimo de ser exhaustivos, estudios más recientes como los de Hecker, Hermenau, Isele y Elbert (2014) en Tanzania, Enosh, Leshem y Buchbinder (2016) en Israel o Umeda, Kawakami, Kessler y Miller (2015) en Japón así lo corroboran.

El castigo físico a los menores ha sido también una práctica habitual en el repertorio de pautas educativas de los padres y madres españoles. Los estudios de prevalencia realizados sobre el tema han mostrado que, para una gran parte de la sociedad, todavía es un método educativo eficaz, aceptable y recomendado, aunque su aceptación haya ido descendiendo en los últimos años (Bussmann, Erthal, y Schroth, 2009).

De hecho, un estudio realizado por el Ministerio de Trabajo y Asuntos Sociales en 1997, en el que se entrevistaba a 3500 españoles mayores de 18 años, señalaba que el 2\% de los españoles aseguraba que pegar es imprescindible "muchas veces" y un 43'3\% indicaa que era imprescindible algunas veces. Un 40’5\% estaba de acuerdo con que "algunas veces es necesario un buen bofetón para mantener la disciplina”. El 30\% reconocía reaccionar de vez en cuando pegándole a su hijo una bofetada ante un conflicto grave (Juste y Morales, 1998).

Posteriormente, los datos recogidos al respecto en el Estudio 2621 del Centro de Investigaciones Sociológicas (CIS, 2005), con la encuesta Actitudes y opiniones sobre la infancia, pusieron de manifiesto que el 59,9\% de la población española considera que "un azote o una bofetada a tiempo puede evitar más tarde problemas más graves".

La normalización del uso del castigo físico como estrategia de disciplina quedó así mismo reflejada en otro trabajo realizado por la organización Save the Children en 2004, en coordinación con la Plataforma de Organizaciones de Infancia y la Dirección General de la Infancia y las Familias del Ministerio de Trabajo y Asuntos Sociales en las comunidades de Castilla la Mancha y Madrid. De este se desprende que el $47 \%$ de las madres y el $46 \%$ de los padres se consideraban con derecho a pegar y gritar a sus hijos.

En el estudio llevado a cabo en 2007 por Bussmann, Erthal, y Schroth (2009), que examinaba la situación en cinco países europeos: Suecia, Austria, Alemania, Francia y España, cinco mil padres (1000 de cada país) fueron entrevistados sobre el uso y las actitudes hacia el castigo físico, sus propias experiencias de violencia y su conocimiento y creencias acerca de la legislación existente. Según este estudio, más de la mitad de los padres españoles (55\%) dijeron haber golpeado levemente a sus hijos en la cara, un $80 \%$ en el trasero, un $31 \%$ reconocieron haberle dado una "sonora" bofetada en la cara y un $6,7 \%$ le había golpeado con un objeto. Aun cuando un $16 \%$ de los padres españoles nunca había usado el castigo físico y un $84 \%$ estaba de acuerdo con que el castigo físico a los menores se debe usar lo menos posible, se trata de cifras muy elevadas, especialmente si se compara con los datos de prevalencia de otros países como Suecia donde la aceptación del castigo físico a los menores no supera el 8\% desde los años noventa (Jason, Jernbro y Langberg, 2012).

Uno de los estudios más reciente realizado en España (Gámez-Guadix et al., 2010b) con estudiantes universitarios de la Comunidad de Madrid refleja que el 63\% de los varones y el 63,8\% de las mujeres fueron castigados físicamente a los 10 años. Las madres utilizaban algo más esta práctica disciplinar que los padres (42,9\% de los padres y 50,1\% de las madres había azotado, abofeteado, golpeado, o dado un manotazo a sus hijos). Por su parte, Calvete, Gámez-Guadix y Orue (2010), en un trabajo con 1371 adolescentes, encontraron que las madres emplean más acciones de disciplina de todo tipo que los 
Análisis de las campañas contra el castigo físico a menores en España...

padres, probablemente porque, a pesar del aumento en los últimos años de la implicación de los padres en la educación de los hijos, estos continúan desempeñando un menor papel que las madres en la crianza de los hijos. Igualmente encontraron un mayor uso de este tipo de disciplina con los hijos varones que con las hijas.

Rosser (2015) realizó una investigación en la misma línea, en la que se pretendía evaluar las prácticas y actitudes existentes en la población en relación con el castigo físico, transcurridos más de 8 años de la entrada en vigor de la ley 54/2007. Para ello se aplicó una adaptación del Inventario de Dimensiones de Disciplina (Discipline Dimensions Inventory, DDI; Straus y Fauchier, 2007), en su versión española para niños y para adultos, a una muestra de 225 menores entre 11 y 18 años de edad de los cuales un 44,9\% eran varones y un 55,1\% mujeres y 96 adultos, padres y madres del alumnado evaluado. Un $77,1 \%$ eran mujeres y el resto, un 22,9\% varones, con una edad media de 44 años $(D T=5,6)$.

Los resultados pusieron de manifiesto una relación entre las experiencias educativas vividas en la infancia y las prácticas educativas que consideran adecuadas como adultos de forma que aquellos padres y madres que sufrieron una disciplina punitiva en su infancia consideraban adecuado aplicarla en la educación de sus hijos.

Por otra parte, los resultados también indicaron un cierto cambio de tendencia puesto que el castigo físico ya no era la estrategia de disciplina parental más habitual a la hora de corregir el comportamiento de los hijos. Esto no parecía ser reflejo de un mayor uso de estrategias de disciplina positiva sino que los adolescentes señalaban haber experimentado con bastante frecuencia prácticas punitivas de índole psicológica, con gritos, descalificaciones, humillaciones o retirada del cariño.

Para la organización Save the Children (2003), la aceptación social del castigo físico es un hecho: "Pegan los hombres, y las mujeres, las personas de distintos medios socioeconómicos o sociales. Las autoridades religiosas, políticas y judiciales se han mostrado a favor del castigo físico en distintas ocasiones". Los argumentos populares que justifican el pegarle a los niños y niñas son variados, desde "todos lo hemos vivido y no nos ha pasado nada" hasta "es un derecho de los padres", como una forma de "encauzar" y hacer fuertes a sus hijos e hijas. Incluso el lenguaje popular habla de "un buen azote", "una buena paliza", entre otras expresiones que justifican el castigo como un buen recurso.

Organismos como Naciones Unidas (1989), Unicef (2002), The Global Initiative to End All Corporal Punishment of Children (2009) o Save the Children (2003) han alertado sobre los riesgos de esta práctica y la necesidad de desarrollar una legislación para su erradicación (Moya y Rosser, 2013).

En Europa sólo 14 países han prohibido explícitamente cualquier castigo corporal. Se trata de Suecia, Finlandia, Noruega, Austria, Letonia, Croacia, Alemania, Bulgaria, Islandia, Ucrania, Rumanía y Hungría, además de Italia y Portugal, cuyos tribunales supremos han declarado ilegal el castigo corporal, aunque no se haya reflejado en la legislación. En concreto en España, hasta 2007, el Código Civil establecía que "los padres podrán (...) corregir razonable y moderadamente a los hijos". Esto era considerado ambiguo desde el punto de vista legal por numerosos expertos y por estos organismos internacionales al sobreentenderse que el derecho de corrección incluía el castigo físico leve.

En consecuencia, Naciones Unidas y el Consejo de Europa instaron a España, así como a los países que no prohibían expresamente el castigo físico a los niños, a que modificaran sus legislaciones para hacerlo. 
Para hacer frente a esta situación, la Ley 54 de 2007 del 28 de diciembre de Adopción Internacional incluyó en su disposición final $1^{\mathrm{a}}$.2. la eliminación del último resquicio legal que permitía utilizar el castigo físico, modificando el artículo 154 del Código Civil que, en su nueva redacción, pasó a señalar que "La patria potestad se ejercerá siempre en beneficio de los hijos, de acuerdo con su personalidad, y con respeto a su integridad física y psicológica”. De esta forma se suprimía expresamente la posibilidad de que los padres corrigieran razonablemente y moderadamente a sus hijos, entendido este derecho de corrección como castigo corporal leve.

Tras los cambios legislativos descritos más arriba, se abrió un amplio debate mediático en España sobre el derecho o no de los padres a pegar a sus hijos para corregir su conducta. En la prensa pudieron leerse numerosos artículos escritos tanto por defensores como por detractores de la medida, así como otros que pretendían aplacar las reacciones.

Como ejemplo del primer caso encontramos titulares como el de El País, (27/06/2007): "La bofetada pierde fuerza”2 en el que se recogen opiniones de expertos que coinciden en señalar que el castigo físico no es nunca una opción válida, o el de El País, (14/12/2007): "El bofetón aún tiene defensores”3 en el que se señala que algunos partidos intentan frenar la prohibición que la ONU había solicitado al Gobierno.

Un año más tarde se podía leer en El País de 4/12/2008 la noticia “45 días de cárcel para una madre que pegó a su hijo por no hacer los deberes”", en el cual se relataba que una mujer había sido condenada a 45 días de prisión y a permanecer alejada a más de 500 metros de su hijo durante un año y 45 días por un delito de malos tratos.

En este caso, la madre fue finalmente indultada, pero estas interpretaciones han provocado un fuerte rechazo social y la crítica de la ciudadanía ante una norma que parecía dejar sin herramientas educativas a los padres y madres.

Los cambios legislativos arriba mencionados no tuvieron el efecto deseado a nivel social, razón por la cual, el artículo 154 del Código Civil fue nuevamente modificado con la redacción de la Ley 26/2015, de 28 de julio, de Modificación del Sistema de Protección a la Infancia y a la Adolescencia que indica que:

"Los hijos no emancipados están bajo la patria potestad de los progenitores. La patria potestad, como responsabilidad parental, se ejercerá siempre en interés de los hijos, de acuerdo con su personalidad, y con respecto a sus derechos, su integridad física y mental" (Art.154).

A nivel normativo, se han ido dando pasos importantes en cuanto a no considerar justificado ni adecuado a derecho el recurso al castigo corporal como medida educativa y en el marco del ejercicio de la patria potestad. Sin embargo, del análisis de casos en España, se desprende que sigue existiendo un sector de la doctrina jurisprudencial que considera plausible enmarcar el castigo corporal leve dentro del "derecho de corrección” como una dimensión del derecho de educación de los

2 Diario El País (27/06/2007): La bofetada pierde fuerza. Disponible en: http://elpais.com/diario/2007/06/26/ sociedad/1182808803_850215.html

3 Diario El País (14/12/2007). El bofetón aún tiene defensores. Disponible en: http://elpais.com/diario/2007/12/14/ sociedad/1197586801_850215.html

4 Diario El País (4/12/2008). 45 días de cárcel para una madre que pegó a su hijo por no hacer los deberes. Disponible en: http://elpais. com/diario/2008/12/05/sociedad/1228431602_850215.html 
progenitores y en el marco de las facultades del ejercicio de la patria potestad. Por su parte, otro sector niega que exista un "derecho de corrección" que de amparo y/o legitime los castigos corporales a menores.

También quedan retos importantes que sortear a nivel socio-cultural pues, como se ha demostrado en otros países Roberts (2000), los cambios de actitudes hacia el castigo físico requieren de otras iniciativas y no es suficiente con los cambios legislativos. Prueba de ello ha sido el hecho de que, la mera alusión a la prohibición sin excepción de todo tipo de castigo corporal a menores, generara cierta alarma en los progenitores. Alarma que tiene su justificación en la tolerancia social, por ejemplo, del cachete y/o la bofetada utilizados como recursos correctivos y/o educativos ante desobediencias e indisciplinas de las y los menores.

Reconociendo que el castigo físico a los menores sigue siendo, una práctica socialmente aceptada (en su grado moderado), es realmente necesario sensibilizar a la sociedad sobre su inconveniencia y, a su vez, dotar a los padres y profesionales de conocimientos suficientes respecto de otras estrategias adecuadas de control y corrección de la conducta.

Una de las formas más efectivas de influir en la conducta es cambiar las actitudes individuales a través de la persuasión (Rucker, Petty y Briñol, (2014). Sin embargo, los medios de comunicación apenas han prestado atención al castigo físico infligido a los niños en comparación con cuestiones como la violencia sexual y la explotación laboral de los niños. Como señala el Consejo de Europa,

"Los medios de comunicación desempeñan un papel fundamental a la hora de forjar opiniones e influir en las normas sociales que inciden a su vez en el comportamiento. La cuestión del castigo físico debería llevarse a la esfera pública; debe crearse un espacio para debatir los problemas y hallar soluciones. Si no hay sensibilización, será difícil lograr una prevención y un cambio de comportamiento duraderos a gran escala" (Consejo de Europa, 2008).

Este ha sido el objetivo del proyecto "PUNISH-ER. Eradication of the use of physical punishment on children within family and institutions" (JUST/2010/DAP3/AG/1337), realizado al amparo de la Iniciativa Daphne de la Unión Europea, en el que participaron Reino Unido, Italia, Alemania, Polonia, Estonia y España y del que la autora de este artículo era investigadora. El proyecto PUNISH-ER pretende contribuir a la erradicación del castigo físico en menores mediante la propuesta de directrices para la elaboración de programas, materiales de sensibilización y materiales formativos, dirigidos a padres y profesionales del sector social y escolar, relacionados con los métodos alternativos al castigo físico.

En concreto, uno de los bloques del proyecto ha sido el análisis de las campañas publicitarias existentes en España sobre el tema, sus fortalezas y debilidades de cara a incrementar el impacto de futuras iniciativas.

En este sentido, la autora de este trabajo participa actualmente, como investigadora principal en España, de un nuevo proyecto europeo, "Hands Up" - Promoting the effective elimination of corporal punishment against children (JUST/2015/ $\mathrm{RDAP} / \mathrm{AG} / \mathrm{CORP})^{5}$. Este nuevo proyecto pretende ahondar en las fortalezas y debilidades que se detectaron en el proyecto Daphne Punish-er, revisando el marco legal, potenciando estrategias de disciplina positiva en padres y madres y desarrollando productos más persuasivos para sensibilizar a la sociedad y acompañar a padres y madres en el proceso de crianza.

5 European Project "Hands Up" - Promoting the effective elimination of corporal punishment against children. Web site: http://www. handsupchildren.org/es/home

$64 \mid n^{\circ}$ 26, pp. 59-80 | doxa.comunicación

enero-junio de 2018 
Como señalan Petty, Briñol y Priester (2009: 125), el éxito de las campañas publicitarias depende en parte de si las comunicaciones trasmitidas son efectivas en el cambio de actitudes de los destinatarios en la dirección deseada y, por otra parte, de si esa modificación de actitudes influye en la conducta de la gente. De ahí la importancia de conocer los procesos responsables del cambio de actitudes a través de los medios de comunicación.

Cualquier variable implicada en el proceso persuasivo (sea del emisor, del mensaje, del receptor, del canal o del contexto) puede afectar a la capacidad y/o motivación del receptor para procesar el mensaje persuasivo, o lo que es lo mismo, la probabilidad de elaboración del receptor (Briñol y Petty, 2006; Briñol, Horcajo, Valle y de Miguel, 2007).

En consecuencia, el objetivo de este trabajo ha sido la revisión de las campañas publicitarias institucionales realizadas en España hasta la fecha para sensibilizar a la población sobre los efectos negativos del castigo físico a los menores. Se analizan las campañas desde que este problema es objeto de estudio, y se atiende, especialmente, a los elementos relacionados con la fuente, el mensaje, el contexto, el canal y el efecto de dichos mensajes.

Tras actualizar el estado de la cuestión se ha procedido al análisis de la información. Se valora si las campañas cumplen con los objetivos de sensibilización y cambio de actitudes planteados en este trabajo para erradicar el uso del castigo físico en la población española y las carencias detectadas.

\section{Método}

\subsection{Búsqueda documental de las campañas de sensibilización institucionales existentes}

Se ha realizado una búsqueda documental de las campañas institucionales realizadas en España contra el castigo físico a los menores, desde la aparición de la primera de ellas en 1999 hasta 2017. La búsqueda dio como resultado el análisis de 6 campañas nacionales de ámbito institucional, además de diversos programas formativos subvencionados por estas para la erradicación de los castigos físicos a los menores a través del desarrollo de estrategias de disciplina positiva (Cuadros 1 a 6). La última de las campañas analizadas corresponde al año 2008 porque no se han emitido campañas institucionales relacionadas con este tema en concreto con posterioridad.

Posteriormente se han realizado las fichas de las campañas, en las cuales se tienen en cuenta la fecha de la campaña, quién la promueve, objetivo, medios de difusión utilizados, metodología utilizada, ámbito, y población a la que se dirige. 
Análisis de las campañas contra el castigo físico a menores en España...

Cuadro 1: Ficha de la campaña Educa no pegues.

\begin{tabular}{|l|l|}
\hline Título: & 1.Educa no pegues \\
\hline Fecha de la campaña: & 1999 \\
\hline Enlace: & https://www.savethechildren.es/publicaciones/educa-no-pegues \\
\hline Quién lo promueve: & $\begin{array}{l}\text { Save the children. UNICEF, CEAPA (Confederación Española de Asociaciones de Padres y Madres } \\
\text { de Alumnos) y CONCAPA (Confederación Nacional Católica de Padres de Alumnos). Con la } \\
\text { colaboración del Ministerio de Trabajo y Asuntos Sociales }\end{array}$ \\
\hline Objetivo: & $\begin{array}{l}\text { Sensibilizar a la población, y particularmente a los padres, madres y profesionales responsables } \\
\text { de la atención a la infancia, sobre las consecuencias asociadas al castigo físico. } \\
\text { Promocionar formas positivas y no violentas de educación y cuidado del niño en el ámbito } \\
\text { familiar. } \\
\text { Informar a los niños y niñas sobre sus derechos. }\end{array}$ \\
\hline Medios de difusión utilizados: & $\begin{array}{l}\text { Acciones en los centros educativos, en las universidades, en los distintos tipos de asociaciones de } \\
\text { infancia y en los medios de comunicación. }\end{array}$ \\
\hline Metodología utilizada: & $\begin{array}{l}\text { Elaboración de materiales de difusión. } \\
-\quad \text { Cursos de formación para padres y madres estructurado en seis sesiones (incluye la } \\
\text { específica sobre la campaña), con los contenidos teóricos y las actividades prácticas } \\
\text { correspondientes. - Guía para padres y madres (primera edición enero 2001). }\end{array}$ \\
\hline Ámbito: & $\begin{array}{l}\text { Difusión de la Convención de los Derechos del Niño. } \\
\text { Promoción de debates públicos, conferencias y seminarios. }\end{array}$ \\
\hline Población a la que se dirige: & $\begin{array}{l}\text { La campaña desarrolla actividades a nivel estatal y autonómico } \\
\text { Padres, profesionales, menores. }\end{array}$ \\
\hline
\end{tabular}

\section{Fuente: Elaboración propia}

Cuadro 2: Ficha de la campaña Corregir no es pegar.

\begin{tabular}{|l|l|}
\hline Título: & 2. Corregir no es pegar \\
\hline Fecha de la campaña: & 2006 \\
\hline Enlace: & https://www.ceapa.es/content/corregir-no-es-pegar-1 \\
\hline Quién lo promueve: & Save the children y Ministerio de Trabajo y Asuntos Sociales. \\
\hline Objetivo: & $\begin{array}{l}\text { Erradicar del castigo físico y de cualquier otra forma de violencia hacia los niños dentro del ámbito } \\
\text { familiar. } \\
\text { Crear espacios de reflexión, formación, debate y apoyo que promuevan formas más sanas y no } \\
\text { violentas de educación hacia los niños. }\end{array}$ \\
\hline Medios de difusión utilizados: & Escuelas de padres, hospitales, servicios sociales \\
\hline
\end{tabular}




\begin{tabular}{|l|l|}
\hline Metodología utilizada: & $\begin{array}{l}\text { Elaboración de trípticos y folletos en los que se ofrecen pautas para abordar el tema del castigo } \\
\text { físico y se presentan alternativas a este tipo de castigo en la educación de los niños en el ámbito } \\
\text { familiar. } \\
\text { Realización de charlas-coloquio para padres y madres, y talleres de formación-debate para los } \\
\text { profesionales. }\end{array}$ \\
\hline Ámbito: & Nacional \\
\hline Población a la que se dirige: & Padres, Profesionales. \\
\hline
\end{tabular}

\section{Fuente: Elaboración propia}

\section{Cuadro 3: Ficha de la campaña Mi bienestar es tu responsabilidad.}

\begin{tabular}{|l|l|}
\hline Título: & 3. Mi bienestar es tu responsabilidad \\
\hline Fecha de la campaña: & 2006 \\
\hline Enlace: & https://www.youtube.com/watch?v=bQqPYPwAjbI \\
\hline Quién lo promueve: & $\begin{array}{l}\text { Federación de Asociaciones para la Prevención del Maltrato Infantil (FAPMI) . Ministerio de } \\
\text { Trabajo y Asuntos Sociales. }\end{array}$ \\
\hline Objetivo: & La concienciación de los ciudadanos sobre cualquier tipo de violencia hacia los menores. \\
\hline Medios de difusión utilizados: & Publicidad en Internet. \\
\hline Metodología utilizada: & Vídeo \\
\hline Ámbito: & Nacional \\
\hline Población a la que se dirige: & Población en general \\
\hline
\end{tabular}

\section{Fuente: Elaboración propia}

Cuadro 4: Ficha de la campaña Mi bienestar es tu responsabilidad. $i$ A que duele?

\begin{tabular}{|l|l|}
\hline Título: & 4. Mi bienestar es tu responsabilidad iA que duele? \\
\hline Fecha de la campaña: & 2007 \\
\hline Enlace: & $\begin{array}{l}\text { Blog: https://todoscontraelmaltratoinfantil.blogspot.com.es/2010/06/02antecedentes-de-la-iii- } \\
\text { campana.html }\end{array}$ \\
\hline Quién lo promueve: & $\begin{array}{l}\text { Federación de Asociaciones para la Prevención del Maltrato Infantil (FAPMI) . Ministerio de } \\
\text { Trabajo y Asuntos Sociales. }\end{array}$ \\
\hline Objetivo: & La concienciación de los ciudadanos sobre cualquier tipo de violencia hacia los menores. \\
\hline
\end{tabular}


Análisis de las campañas contra el castigo físico a menores en España...

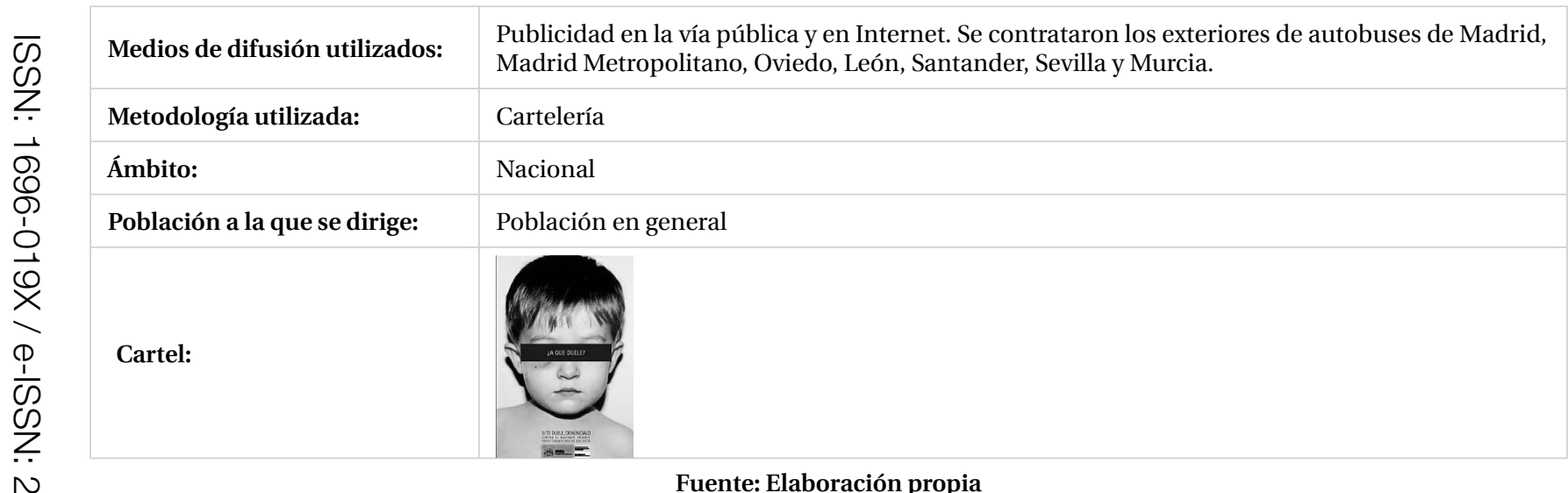

Cuadro 5: Ficha de la campaña Mi bienestar es tu responsabilidad $i^{A}$ que duele?

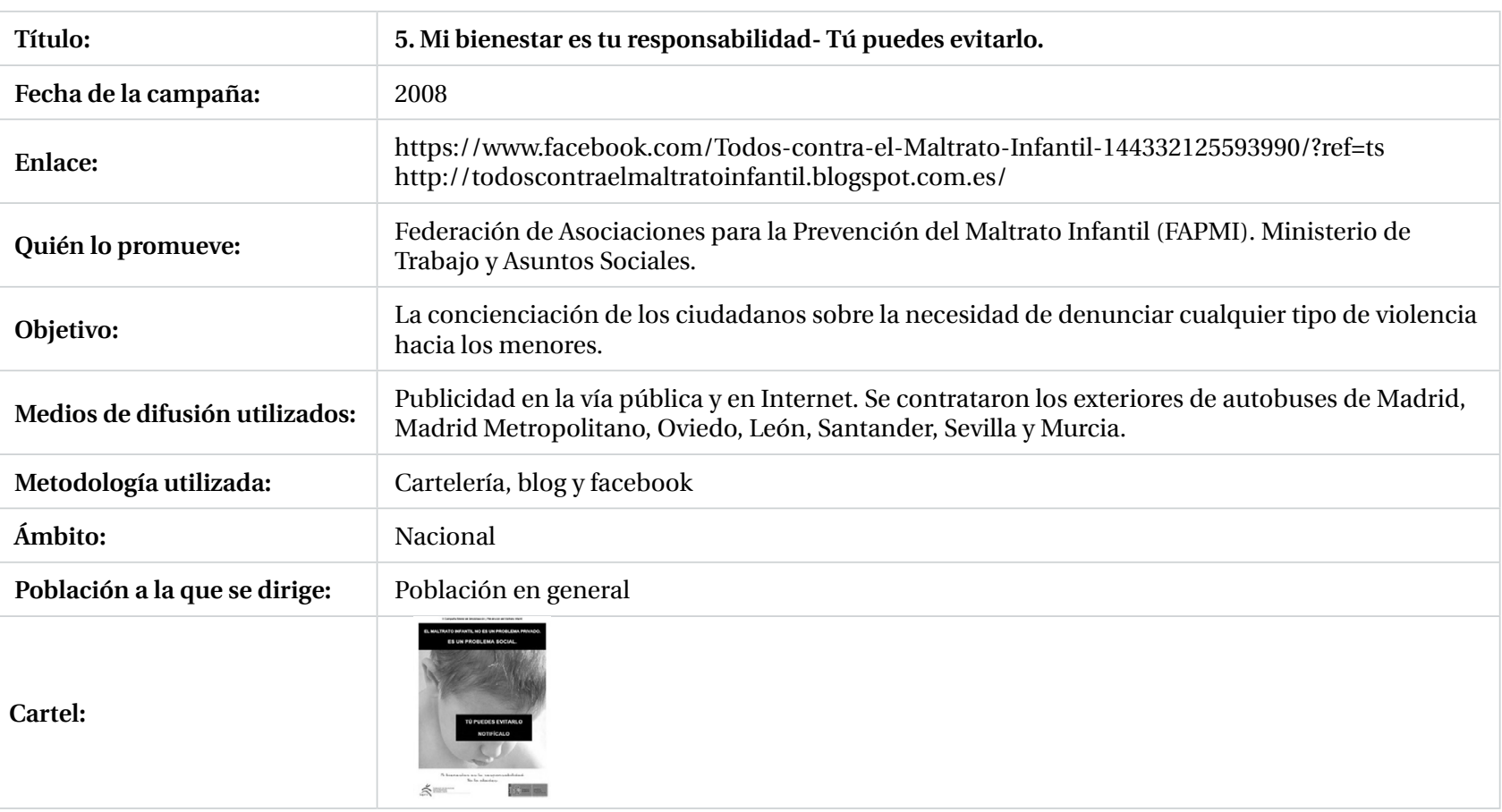

Fuente: Elaboración propia

$68 \mid n^{\circ} 26$, pp. 59-80 | doxa.comunicación

enero-junio de 2018 
Cuadro 6: Ficha de la campaña Levanta la mano contra el castigo físico. Tus manos son para proteger.

\begin{tabular}{|c|c|}
\hline Título: & 6. Levanta la mano contra el castigo físico / Tus manos son para proteger \\
\hline Fecha de la campaña: & 2008 \\
\hline Enlace: & Spot www.youtube.com/watch?v=248AQHLSqTo \\
\hline Quién lo promueve: & Consejo de Europa y Ministerio de Educación, Política Social y Deporte. \\
\hline Objetivo: & $\begin{array}{l}\text { Sensibilizar sobre la necesidad de suprimir las técnicas de educación basadas en el castigo } \\
\text { físico, creando una consciencia en los padres, educadores y a los adultos en general, de que los } \\
\text { niños tienen derechos, y no se les deben someter a conductas como bofetadas, cachetes, azotes, } \\
\text { gritos, vejaciones, etc., que ningún adulto toleraría. }\end{array}$ \\
\hline Medios de difusión utilizados: & Televisión. Internet \\
\hline Metodología utilizada: & Spot publicitario “Tus manos son para proteger”, Cartelería. \\
\hline Ámbito: & Nacional, Europeo \\
\hline Población a la que se dirige: & Población en general, padres \\
\hline Cartel: & 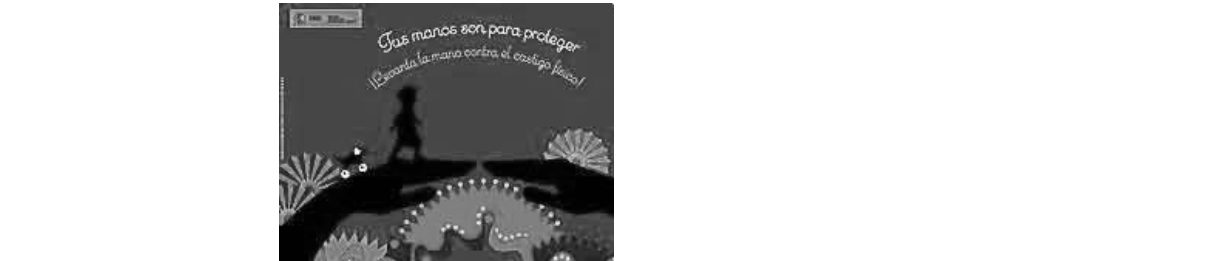 \\
\hline
\end{tabular}

Fuente: Elaboración propia

\subsection{Análisis de contenido categorial de los recursos publicitarios utilizados}

Se ha realizado, siguiendo a autores como Vázquez Sito (1997), Andreu (2002) u Oliveira (2008), un análisis de contenido categorial de los materiales y vídeos de las campañas.

El análisis de contenido categorial temático consiste en tratar de ir identificando los elementos de los mensajes publicitarios para, posteriormente, proceder a su agrupamiento en categorías donde se consideran las similitudes o semejanzas que existan entre éstas en función de criterios preestablecidos según los objetivos de investigación. Un anuncio publicitario puede tener presente una o ninguna de las variables por lo tanto se expresa la cantidad de veces que aparece una variable sobre el total de campañas analizadas.

Atendiendo al objetivo del estudio, las categorías se han construido tratando de responder a las cuestiones que permiten definir un modelo adecuado para describir un determinado acto de comunicación. En concreto, ¿Quién?; ¿Qué y cómo se dice?; ¿ Por qué canal lo dice?; ¿A quién?; ¿Con qué efecto?. Y sobre las variables, señaladas como elementos claves de la comunicación persuasiva (Briñol et al., 2007; Moya, 2000): 
Análisis de las campañas contra el castigo físico a menores en España...

\section{QUIÉN LO DICE}

\subsection{Promotores}

1.2. Personajes presentes en la comunicación: las víctimas, los protectores, castigadores, los expertos.

1.3. Narrador: se dividieron en dos categorías.

1.3.1. El narrador en tercera persona: cuando es impersonal, conocido como "voz en off. La "voz" del narrador puede ser un texto.

1.3.2.Personajes: cuando son las personas que aparecen en la comunicación quienes hablan (bien en grabaciones o recogiendo sus testimonios en textos).

2. QUÉ DICE

2.1. ¿Qué se comunica?: El objetivo de la campaña.

2.2. ¿Cómo se comunica? (elementos persuasivos del mensaje):

2.2.1. Las conclusiones son claras y explícitas o por el contrario ambiguas, teniendo el público que sacar sus propias conclusiones.

2.2.2. Los mensajes son unilaterales, sólo con argumentos de apoyo a la posición defendida por el comunicador, o bilaterales, que contiene puntos de vista contrarios a este

2.2.3. Organización del mensaje. Los mejores argumentos van al principio (Primacia) o al final (Recencia).

2.2.4. Probabilidad de ocurrencia de las consecuencias contenidas en el mensaje.

2.3. Tono de comunicación: Se refiere a las diferentes perspectivas desde la narrativa con las que se puede elaborar un discurso, la forma de comunicar el mensaje.

2.3.1. Emotivo: existe una alusión a escenarios o conductas afectivas con las cuales las personas se pueden ver identificadas. Apela a los sentimientos y a las emociones.

2.3.2. Informativo: su característica principal es informar, mostrar objetivamente un hecho o situación.

3. CANAL

3.1. Medios de difusión utilizados: TV, Radio, Prensa, etc.

\section{A QUIÉN LO DIRIGE}

4.1. Destinatarios: Menores, Padres, Profesionales, Sociedad en general, Administración.

\section{EN QUÉ CONTEXTO SE PRODUCE}

5.1. Si responde a episodios ocurridos coincidiendo con su elaboración

\section{CON QUÉ EFECTO}

\subsection{Memoria de actividades}

6.2. Impacto en redes sociales: información sobre el número de visitas recibidas en Internet por los vídeos y/o materiales de las campañas

Los resultados para los diferentes tipos de soporte se han unificado. Se valoran como l=sí; $0=$ no y se contabilizan en un cuadro donde aparecen los totales de las frecuencias de cada una de las variables estudiadas. 


\section{Resultados}

A partir del análisis descriptivo original realizado por el equipo de España del proyecto PUNISH-ER, y con los nuevos datos recopilados en este trabajo, se procedió a la cuantificación de estos parámetros, de forma que se pudieran visualizar tendencias que se presentan en las campañas y que posteriormente se analizan (Cuadro 7).

Cuadro 7. Análisis de contenido categorial de los recursos persuasivos utilizados en las campañas.

\begin{tabular}{|c|c|c|c|c|c|c|c|c|c|}
\hline & Campaña & & 1 & 2 & 3 & 4 & 5 & 6 & Total \\
\hline \multirow{6}{*}{ QUIÉN: } & \multirow{4}{*}{ Personajes } & Victimas (los menores) & 1 & 1 & 1 & 1 & 1 & 1 & 6 \\
\hline & & Adultos Agresores (los progenitores) & & & 1 & & 1 & & 2 \\
\hline & & Adultos protectores & & & 1 & & & & 1 \\
\hline & & Expertos & & & & & & & 0 \\
\hline & \multirow{2}{*}{ Tipos de texto } & Narración institucional & 1 & 1 & 1 & 1 & & 1 & 5 \\
\hline & & Personajes & & 1 & & & & & 1 \\
\hline \multirow{13}{*}{ QUÉ: } & \multirow{4}{*}{ Objetivo de la campaña } & Evitar bofetada, cachete & & & 1 & & & & 1 \\
\hline & & Invita a la denuncia & & & & 1 & 1 & & 2 \\
\hline & & Promocionar otra forma de educar & 1 & 1 & & & & 1 & 3 \\
\hline & & Informar consecuencias & 1 & 1 & & & & & 2 \\
\hline & \multirow{7}{*}{ Contenido } & Conclusiones explícitas & 1 & 1 & & 1 & & & 3 \\
\hline & & Conclusiones implícitas & & & 1 & & & 1 & 2 \\
\hline & & Mensajes unilaterales & 1 & 1 & & 1 & 1 & 1 & 5 \\
\hline & & Mensajes bilaterales & & & 1 & & & & 1 \\
\hline & & Primacía & 1 & 1 & & & & & 2 \\
\hline & & Recencia & & & 1 & 1 & 1 & 1 & 4 \\
\hline & & Probabilidad de ocurrencia & 1 & 1 & & & 1 & 1 & 4 \\
\hline & \multirow{2}{*}{ Tono } & Emotivo & & & 1 & & & 1 & 2 \\
\hline & & Informativo & 1 & 1 & & & & 1 & 3 \\
\hline \multirow{5}{*}{ A QUIÉN: } & \multirow{5}{*}{ Destinatarios } & Niños & 1 & & & 1 & & & 2 \\
\hline & & Padres & 1 & 1 & 1 & & & 1 & 4 \\
\hline & & Profesionales & 1 & 1 & & & & & 2 \\
\hline & & Administración & & & & & & & 0 \\
\hline & & Sociedad en general & & & 1 & 1 & 1 & 1 & 4 \\
\hline
\end{tabular}


Análisis de las campañas contra el castigo físico a menores en España...

\begin{tabular}{|c|c|c|c|c|c|c|c|c|c|}
\hline \multirow{9}{*}{ CANAL: } & \multirow{9}{*}{ Medios de difusión } & T.V & & & & & & 1 & 1 \\
\hline & & Prensa & & & & 1 & & 1 & 2 \\
\hline & & Publicidad exterior & & & & 1 & & & 1 \\
\hline & & Internet & 1 & 1 & 1 & 1 & 1 & 1 & 6 \\
\hline & & Correo directo & & & & & & & 0 \\
\hline & & Impresos & 1 & 1 & & 1 & & 1 & 4 \\
\hline & & Exposiciones & & & & 1 & & & 1 \\
\hline & & Acciones educativas & 1 & 1 & & & & & 2 \\
\hline & & Material didáctico & 1 & & & & & & 1 \\
\hline \multirow{2}{*}{ EFECTO: } & \multirow{2}{*}{ Evaluación impacto } & Memoria de actividades & & & & 1 & & & 1 \\
\hline & & Estudios prevalencia & & & 1 & & & & 1 \\
\hline
\end{tabular}

Fuente: Elaboración propia a partir del estudio elaborado por el equipo investigador del proyecto europeo PUNISH-ER en España (Dominici, Henao y Rico (coords.), 2013)

Del análisis de las campañas efectuadas en España en aras a la erradicación del castigo físico se pueden concluir diferentes cuestiones, tanto sobre su contenido como sobre su impacto.

La temporalización de las campañas muestra un creciente interés por el tema en los años próximos a la modificación legal del 2007, para luego desaparecer de los objetivos del gobierno a partir de 2008.

En el Cuadro 7 se aprecia que, en la mayoría de los casos, el emisor es el gobierno junto a entidades de reconocido prestigio internacional, quienes, mediante voz en off o textos escritos, denuncia y/o induce a la acción (5 de las 6 campañas analizadas). Esta estrategia va a conferir credibilidad al mensaje y facilita su aceptación.

Los protagonistas son en todos los casos los propios menores, las víctimas (5 de las 6 campañas), aunque también aparecen en ocasiones los agresores y en algún caso los adultos con rol protector. Eso hace que se pongan de manifiesto los perjuicios del castigo físico pero no las razones de su aplicación ni las estrategias alternativas.

En los lemas que reflejan la intención del mensaje destacan (3 de las 6 campañas) los mensajes positivos y la invitación a usar estrategias educativas alternativas al castigo físico ("Educa, no pegues", "Tus manos son para proteger"). Así mismo aparece la conveniencia de evitar los métodos de disciplina física como la bofetada o el cachete.

Es importante el peso de otras estrategias orientadas a informar de las consecuencias del castigo físico en los menores y de fomentar que la ciudadanía denuncie estos hechos (4 de las 6 campañas).

Respecto a los tipos de mensajes utilizados en las campañas, el Cuadro 7 muestra un predominio de mensajes unilaterales (en 5 de las 6 campañas) y con conclusiones explícitas (en 3 de las 6 campañas). En principio estas estrategias permitirían asegurar la atención de un público menos motivado y con menos habilidades. Pero precisamente la campaña "Tus manos son para proteger" que ha sido la de mayor alcance, por servirse de un medio de difusión masivo como es la televisión utiliza conclusiones implícitas y por tanto de más difícil comprensión para el público en general. 
Los mejores argumentos suelen aparecer al final del mensaje (en 4 de las 6 campañas) lo que parece favorecer la comprensión del mensaje si los potenciales receptores están poco motivados.

Por otra parte, la mayoría (4 de las 6 campañas) informan de la alta probabilidad de ocurrencia de consecuencias positivas si se siguen las recomendaciones del mensaje.

Finalmente, predominan los tonos informativos (en 3 de las 6 campañas analizadas) frente a los emotivos. Como se trata de cambiar las creencias que sostiene las actitudes favorables al uso del castigo físico, esta estrategia persuasiva parece en principio adecuada aunque, su uso podría estar condicionado por el soporte en papel de muchos de los materiales.

Las campañas se dirigen mayoritariamente a padres y madres (en 4 de las 6 campañas), y en menor medida a los propios menores para que sepan reconocer y defender sus derechos (en 2 de las 6 campañas), y a los profesionales (en 2 de las 6 campañas) para orientar su intervención.

Así mismo se observa que, mientras las primeras campañas emitidas tenían un claro contenido didáctico orientado a padres y menores, las más recientes pretender sensibilizar a la población en general con el problema.

Atendiendo a la forma de difusión, sólo dos de ellas tuvieron su difusión mediante una cuña televisiva, dirigiéndose las otras más a entornos educativos y cívicos, mediante folletos (4 de las 6 campañas), actividades y manuales didácticos (3 de las 6 campañas), publicidad exterior (en 1 de ellas), e incluso en un caso una exposición con los dibujos de los menores. Internet se ha hecho eco de todas ellas, difundiendo los vídeos a través de Youtube, los materiales a través de las webs y blogs y también a través de redes sociales como Facebook.

Respecto al contexto en el que se emitieron, las campañas de 2006 tuvieron como precedente el estudio del CIS antes mencionado y las dos últimas se difundieron tras la entrada en vigor de la Ley 54 de 2007 del 28 de diciembre, que prohibía utilizar el castigo físico. Las nuevas modificaciones legislativas de 2015 no han llevado consigo ninguna nueva campaña institucional.

El análisis de impacto de las campañas estudiadas es complicado ya que desde 2005 que no se cuenta con estudios de prevalencia de esta práctica disciplinaria que permitan valorar un hipotético descenso en el uso del castigo físico por su influencia. Tampoco existe una memoria de actividades relacionadas con la implantación de las campañas salvo en un caso, la campaña de 2007. Sin embargo, las reacciones en contra que provocó en la población la entrada en vigor de la Ley 54 de 2007 del 28 de diciembre, que prohibía utilizar el castigo físico, llevan a pensar en el bajo nivel de concienciación social sobre los perjuicios de esta práctica.

Ante la falta de una evaluación del impacto por parte de las instituciones promotoras, en el Cuadro 8 se ha optado por considerar, a partir de la herramientas como Woorank (www.woorank.com) o Website seo analysis (https://iwebchk.com), determinados indicadores como el número de visitas recibidas en las webs, vídeos y/o redes sociales relacionadas con estas (View per cannel o VPC), el número de seguidores en Twiter o compartidos en Facebook. 
Análisis de las campañas contra el castigo físico a menores en España...

\begin{tabular}{|c|c|c|c|}
\hline \multicolumn{4}{|c|}{ Cuadro 8: Análisis impacto en internet. } \\
\hline Nombre de la campaña: & VPC & Seguidores en twiter & Compartido en Facebook \\
\hline Educa no pegues: & 13267 & & 973 \\
\hline Corregir no es pegar: & 913 & 6.208 & 178 \\
\hline Mi bienestar es tu responsabilidad: & 346 & & \\
\hline Mi bienestar es tu responsabilidad. ¿A que duele?: & \multirow{2}{*}{1579} & & 14 \\
\hline Mi bienestar es tu responsabilidad. Tú puedes evitarlo: & & & 1989 \\
\hline Tus manos son para proteger: & 253.076 & & 478 \\
\hline
\end{tabular}

\section{Fuente: Elaboración propia}

Destaca, con diferencia, la última de las campañas en cuanto el seguimiento en la población, debido probablemente al hecho de haber elegido un medio de difusión masivo como es la televisión y utilizar elementos atractivos como la música y el color. Por el contrario, su impacto puede haber estado limitado por otros factores que pueden haber impedido la elaboración del mensaje como su ambigüedad.

\section{Discusión}

Erradicar el uso del castigo físico en la educación de los hijos está resultando una tarea ardua. Aunque las cifras de prevalencia parecen haber descendido en muchos países en la última década (Zolotor et al., 2011), una mirada a las reacciones que provocó el cambio legislativo de 2007 en nuestro país, dirigido a prohibir el uso del castigo físico como medida de disciplina parental, así como los últimos estudios realizados al efecto (Calvete, Gámez-Guadix y Orue, 2010; Gámez-Guadix et al., 2010b) indican hasta qué punto existe una tolerancia social hacia este como forma de corrección a los hijos dentro de la familia y una discrepancia entre las creencias y las conductas parentales. Aunque también muestra como el cambio de tendencia no se dirige al desarrollo de estrategias de disciplina positiva sino a la sustitución del castigo físico por otras estrategias punitivas más vinculadas al maltrato emocional como insultar, amenazar, humillar, etc. (Rosser, 2015).

Tampoco se han realizado nuevas encuestas a nivel nacional y el tema ha desaparecido de los barómetros sociológicos.

Sin embargo, la investigación ha puesto de manifiesto los riesgos que conlleva esta práctica educativa en el desarrollo psicosocial de los menores (Fergusson y Lynskey, 1997; Gámez-Guadix et al., 2010a; Grogan-Kaylor, 2004; Landsford et al., 2014; Scott, Lewsey, Thompson y Wilson, 2013: Simons y Wurtele, 2010; Smith, Springer y Barret, 2011; Torío, Peña e Inda, 2008, entre otros).

A la evidencia científica sobre los perjuicios de esta práctica educativa se une el empeño de organismos internacionales de prestigio como UNICEF o Save the Children para su erradicación y el desarrollo de estrategias de disciplina positiva, junto al mandato legal propiciado por los últimos cambios legislativos en materia de protección a la infancia implantados en España. 
Pero, lo que podría resultar un marco propicio para el desarrollo e impacto de estas campañas, ha chocado con las actitudes de la población, donde perviven unas creencias y costumbres muy arraigadas en la sociedad sobre la "utilidad" del cachete o la bofetada como mecanismo de corrección, y con la alarma social provocada por los cambios normativos.

El hecho de que no se trate de un tema prioritario para la población a la que se dirigen estas campañas, junto a la polémica y/o falta de acuerdo manifestada por los responsables de velar por el cumplimiento de las normas legales sobre lo que se debe y no se debe hacer en el ámbito de la educación a los hijos, se convierte en una amenaza más que puede estar incidiendo en la escasa presencia de campañas sobre este tema en los medios de comunicación.

Así las cosas, resta evaluar cómo son y cómo han funcionado las campañas existentes para persuadir a la sociedad de la utilización del castigo físico en España.

Cambiar los hábitos y actitudes de los individuos es difícil (Briñol et al., 2007), pero la publicidad puede desarrollar estrategias persuasivas que faciliten este cambio de actitudes mediante campañas de sensibilización.

Para lograr este cambio actitudinal y que las campañas se conviertan en un mecanismo de prevención primaria, a través de la sensibilización, deben ir dirigidas a la sociedad en general, y lanzarse a través de medios de comunicación masivos que permitan crear estados de opinión contrarios al uso del castigo físico como estrategia educativa.

Los resultados de este trabajo confirman, en primer lugar que, efectivamente, y como denunciaba el Consejo de Europa (2008), los medios de comunicación apenas han prestado atención al castigo físico infligido a los niños. Este tema sólo cobra cierto protagonismo en España entre los años 2006 y 2008, cuando este empeño exige una acción sostenida en el tiempo para tener efectos (Comité sobre los Derechos del Niño, Observación General No. 8, párrafos 45-49).

El cambio de las actitudes profundamente enraizadas lleva tiempo y exige una inversión sostenida en actividades a diversos niveles, por lo que campañas de este tipo deberían tener una presencia mayor y más continuada en los medios. Como señalan Benet y Nos Aldas (2003), "la publicidad social debe seguir un proceso largo y profundo para modificar las creencias, las ideologías y cambiar las actitudes a largo plazo”. La revisión de las campañas objeto de este trabajo muestra sin embargo un bajo nivel de difusión en medios de comunicación masivos como la televisión y escasa continuidad.

Por otra parte, vender intangibles como la educación positiva o la no violencia puede convertirse en una difícil tarea si no se tienen motivaciones claras que despierten el interés de la comunidad (Orozco, 2010), y el análisis de las reacciones que suscitaron en su día los cambios legislativos, o los datos del CIS (2005) no reflejan que esta motivación exista en la sociedad española. De este estudio se desprende que las campañas emitidas tampoco han tenido gran presencia en los medios.

Si nos ceñimos al resultado de las investigaciones llevadas a cabo en nuestro país, su impacto también ha sido escaso en el cambio actitudinal de la población (Calvete, Gámez-Guadix y Orue, 2010; Gámez-Guadix et al., 2010b), o ha virado hacia otro tipo de prácticas punitivas de corte más emocional (Rosser, 2015).

Si el escenario para que los mensajes contra el castigo físico calen en la población cuenta con estos obstáculos, se hace imprescindible contar con estrategias persuasivas potentes para aspirar a un cambio de actitudes en la sociedad.

En este sentido, sabemos que, para que las campañas publicitarias de tipo social resulten eficaces en el desarrollo de sus objetivos debe estar claro qué se desea decir (el mensaje), el objetivo de la comunicación (porqué y con qué finalidad) y el 
público al que va dirigida la comunicación (Orozco, 2010). La elección de los elementos como la fuente, el tipo de mensaje, etc., vendrán determinados por el tipo de audiencia (Moya, 2000; Morales, Moya, Gaviria. y Cuadrado, 2007).

Para avanzar en la erradicación del castigo físico moderado sobre los menores, las campañas objeto de este trabajo deberían informar a los padres y madres que lo utilizan de sus perjuicios y, a través de sus mensajes, persuadirles para que cambien de estrategia educativa. Es importante, por tanto, conocer al público objetivo (Moliner, 1998; Orozco, 2010) lo que supone, en el tema que nos ocupa, conocer las razones por las cuales los padres de familia recurren al castigo corporal y, a partir de ahí, establecer objetivos y la manera de lograrlos, para poder preparar los materiales y herramientas necesarios para promover la crianza positiva (Iniciativa global para acabar con todo castigo corporal hacia niños y niñas y Save the children, 2010).

Las campañas de publicidad social pueden modificar actitudes incluso profundamente arraigadas, actuando directamente sobre las creencias; es decir aportando información adicional de forma que se modifique la opinión del espectador (por ejemplo, ampliando o profundizando la visión que tenía anteriormente de un problema) u ofreciendo nuevos puntos de vista para que, al cambiar la perspectiva de enfoque, también cambie la valoración del problema (Condeluppi, 2007).

Sabemos que, generalmente los padres y madres que utilizan el castigo físico lo hacen porque es lo que vieron hacer a sus mayores y porque con ello pretenden educar y corregir las conductas del/de la menor, por lo que las campañas deberán cuestionar estas creencias tan arraigadas y mostrar que se puede conseguir lo mismo con otros métodos.

Sin embargo, un análisis crítico de los contenidos de las campañas estudiadas indica que los mensajes estudiados pretenden suscitar emociones en los espectadores (componente afectivo). “A que duele?”, y cambiar la forma de actuar (componente conativo) de las víctimas (“Educa, no pegues”), o de la sociedad (“Tu puedes evitarlo”), para cambiar sus actitudes, pero no inciden en las razones que llevan a los progenitores a utilizar el castigo físico como herramienta educativa, ofreciendo otros puntos de vista que muestren las ventajas o beneficios de sustituir estas prácticas por una disciplina positiva.

No se incide, por tanto, en el componente cognitivo de las actitudes que se desea cambiar. Se recurre más bien a estrategias de clave heurística, imágenes llamativas, personajes atractivos, mensajes unilaterales, conclusiones explícitas, etc. que permiten llegar a un público poco implicado, pero sin que apenas generen pensamientos en respuesta a la propuesta. Por lo tanto, producirán un cambio de actitudes más débil en cuanto a resistencia, estabilidad, y predicción de la conducta futura (Briñol et al., 2007).

Finalmente hay que destacar que, no basta con concienciar a la población sobre la prohibición. Es necesario educar sobre las consecuencias negativas del castigo corporal a los niños, pero dando alternativas educativas y haciendo una promoción intensa de los métodos disciplinarios positivos, no violentos y participativos (Iniciativa global para acabar con todo castigo corporal hacia niños y niñas, 2009).

En este sentido, algunas de las campañas analizadas en este trabajo proponen estrategias para desarrollar otro tipo de pautas de educativas mediante manuales, talleres de formación, etc. Sin embargo, no lo hacen respondiendo a una demanda social. Esto pone en riesgo su efectividad ya que, sólo cuando ya se haya generado un cierto interés y preocupación por el tema es cuando pueden ser efectivas otro tipo de estrategias más dirigidas a población específica (prevención secundaria), mediante materiales didácticos, charlas, talleres, etc. dirigidas a profesionales, padres, y a los propios menores. 
Para concluir, hay que señalar que, para lograr el cambio actitudinal, es necesario potenciar la emisión de campañas de sensibilización contra el castigo físico a los menores como estrategia para corregir sus conductas pero, además, es importante hacerlo atendiendo a los procesos psicológicos que van a propiciar un cambio en las actitudes fuerte y duradero que genere, a su vez, cambios en las conductas y permita su erradicación.

En consecuencia, se sugiere que, para diseñar estas campañas se parta de un proceso de investigación previa donde se contemple el estudio del público objetivo, de los lugares donde se va a llevar a cabo la campaña y de los medios de comunicación a utilizar, para plantear unos objetivos adecuados y una correcta relación con las estrategias y las tácticas (Orozco, 2010). Por otra parte, los medios de comunicación, deberían asumir un rol más activo en la defensa de los derechos de la infancia, tal y como promueve su Código de autorregulación de contenidos televisivos e infancia cuando señala, entre sus objetivos, el de fomentar que los presentadores o conductores de programas en directo adviertan las situaciones que puedan afectar a la protección de los menores, de forma que se minimicen los eventuales perjuicios que puedan causarles (García Galera, 2008). Igualmente se sugiere que, tanto desde el ámbito publicitario, como también en el educativo y el legal, deberían desarrollarse todo un elenco de actuaciones encaminadas a deslegitimar el castigo físico como estrategia educativa y buscar alternativas para la crianza de los hijos/as.

\section{Referencias bibliográficas}

Andréu, J. (2002): Las técnicas de análisis de contenido: una revisión actualizada. Fundación Centro Estudios Andaluces, Universidad de Granada, 10(2), pp. 1-34.

Baumrind, D. (1971): “Current patterns of parental authority”, Developmental Psychology, n.4, pp.1-103.

Briñol P; Horcajo, F. J.; Valle, C. y de Miguel, J. M. (2007): “Cambio de actitudes a través de la comunicación”, en Morales, J. F., Gaviria, E., Moya Morales, M. C. y Cuadrado, M. I. (coords.): Psicología social. Madrid: MacGraw Hill, pp. 491-516.

Briñol, P. y Petty, R. E. (2006): "Fundamental Processes Leading to Attitude Change: Implications for Cancer Prevention Communications”, Journal of communication, vol. 56, n. S1, pp. S81-S104.

Bunting, L.; Webb, M. A. y Healy, J. (2010): “In Two Minds? Parental Attitudes toward Physical Punishment in the UK”, Children and society, vol. 24, pp. 359-370.

Bussmann, K.D.; Erthal, C y Schroth, A. (2009): “The Effect of Banning Corporal Punishment in Europe: A Five-Nation Comparison”. Germany: Martin-Luther-Universität Halle-Wittenberg.

Calvete, E.; Gámez-Guadix, M. y Orue, I. (2010): “El Inventario de Dimensiones de Disciplina (DDI), Versión niños y adolescentes: Estudio de las prácticas de disciplina parental desde una perspectiva de género”, Anales de psicología, vol. 26, n. 2, pp. 410-418.

Centro de Estudios Sociológicos (2005): “Actitudes y opiniones sobre la infancia”. Estudio no 2621.

Comité de los Derechos del Niño, de las Naciones Unidas (2006): Observación general. n. 8.

Codeluppi, V. (2007): “El papel social de la publicidad”, Pensar la publicidad, vol.1, n.1, 152. 
Análisis de las campañas contra el castigo físico a menores en España...

Consejo de Europa (2008): Abolición del castigo físico infligido a niños y niñas. Preguntas y respuestas. Strasbourg: Council of Europe.

Dietz, T. L. (2000): “Disciplining children: characteristics associated with the use of corporal punishment", Child Abuse and Neglect, vol. 12, pp. 1529-1542.

Dominici, G;, Henao, A. y Rico, R. (coords.) (2013): Erradicación del uso del castigo físico en la familia y en las instituciones. Campañas de sensibilización. Alicante: Asociación Altea-España.

Enosh, G., Leshem, E. y Buchbinder, E. (2016). Attitudes Toward Domestic Violence and Corporal Punishment Among Former Soviet Union Immigrants in Israel. Violence against women, 22(11), pp. 1326-1342.

Fauchier, A. y Straus, M. A. (2007): Dimensions of discipline by fathers and mothers as recalled by university students. Durham, NH: Family Research Laboratory, University of New Hampshire.

Fergusson, D. M. y Lynskey, M. T. (1997): “Physical punishment/maltreatment during childhood and adjustment in young adulthood”, Child abuse and neglect, vol.7, pp. 617-630.

Fréchette, S., y Romano, E. (2015): Change in corporal punishment over time in a representative sample of Canadian parents. Journal of Family Psychology, 29(4), pp. 507-517.

Gámez-Guadix, M.; Straus, M.; Carrobles, J. A.; Muñoz-Rivas, M. y Almendros, C. (2010a): “Corporal punishment and longterm behavior problems: The moderating role of positive parenting and psychological aggression”, Psicothema vol. 22, n. 4, pp. 529-536.

Gámez-Guadix, M.; Orue, I. ; Calvete, E.; Carrobles, J. A. y Muñoz-Rivas, M. (2010b): “Psychometric properties of the Spanish version of the Dimensions of Discipline Inventory (DDI) in university students”, Psicothema, vol. 22, n. 1, pp.151-156.

García Galera, M. C .(2008): “Los derechos de la infancia frente al televisor. ¿Quién los defiende?”, Doxa comunicación, vol. VI, pp. 93-111.

Gershoff, E. T. (2002): “Corporal punishment by parents and associated child behaviors and experiences: A meta-analytic and theoretical review”, Psychological Bulletin, vol. 128, pp. 539-579.

Grogan-Kaylor, A. (2004): “The effect of corporal punishment on antisocial behavior in children”, Social Work Research, vol. 28, n. 3, pp. 153-162.

Hecker, T., Hermenau, K., Iseele, D. y Elbert, T. (2014): Corporal punishment and children's externalizing problems: a cross-sectional study of Tanzanian primary school aged children. Child abuse and neglect, 38(5), pp. 884-892.

Hodgkin, R. y Newell, P. (2002): Manual de Implementación de la Convención sobre los Derechos del Niño (2a. ed.). Genève: UNICEF.

Holden, G. W., Hawk, C. K., Smith, M. M., Singh, J. y Ashraf, R. (2017): Disciplinary Practices, Metaparenting, and the Quality of Parent-Child Relationships in African-American, Mexican-American, and European-American Mothers. International journal of behavior and development, 41(4), pp. 482-490. 
Iniciativa global para acabar con todo castigo corporal hacia niños y niñas (2009): "Prohibir el castigo corporal de los niños. Guía sobre la reforma legal y otras medidas". Londres: The Association for the Protection of All Children.

Iniciativa global para eliminar toda forma de castigo corporal hacia los niños y niñas y Save the Children Suecia (2010): "Manual de campañas. Eliminando el castigo corporal y otros castigos crueles y degradantes hacia los niños y niñas mediante la reforma legal y el cambio social". London: The Association for the Protection of All Children.

Jason, S., Jernbro, C. y Langberg, B. (2012): Corporal punishment and other humiliating behaviour towards children in Sweden - a national study. Karlstad: Stiftelsen Allmänna Barnhuset and writers.

Juby, C. (2009): Parental attitude: a mediating role in disciplinary methods used by parents. Child and adolescent social work journal, 26, pp. 519-531.

Juste, M. G. y Morales, J. M. (1998): “La violencia hacia los hijos en el ámbito familiar: lo que opinan los españoles”, Estudios de Juventud, vol.42, pp. 35-46.

Khoury-Kassabri M, Attar-Schwartz S, y Zur H. (2014): Understanding the mediating role of corporal punishment in the association between maternal stress, efficacy, co-parenting and children's adjustment difficulties among Arab mothers. Child abuse and neglect, 38(6), pp. 1073-1082.

Lansford J. E., et al., 2014). Corporal punishment, maternal warmth, and child adjustment: a longitudinal study in eight countries. Journal of clinical children and adolescent psychology,43(4): pp. 670-685.

Ley 54/2007, de 28 de diciembre, de Adopción Internacional. BOE de 29 de diciembre de 2007. BOE 29 de diciembre de 2007.

Ley 26/2015, de 28 de julio, de Modificación del Sistema de Protección a la Infancia y a la Adolescencia. BOE 29 de julio de 2015.

Moliner, M. A. (1998): Marketing social. La gestión de las causas sociales. Madrid: ESIC.

Morales, J. F., Moya, M., Gaviria, E. y Cuadrado, I. (2007). Psicología social (3a. ed.). Madrid: Mc Graw-Hill.

Moya, M. (2000): “Persuasión y cambio de actitudes”, en: Morales, J. F. y Huici, C. (oords.): Psicología social (pp. 153-170). Madrid: UNED.

Moya, C. y Rosser, A. (2013): “Análisis legislativo del uso del castigo físico en el ámbito familiar”. En: VV. AA.: Erradicación del uso del castigo físico en la familia y en las instituciones. Legislación y prevalencia. Alicante: Asociación Altea-España, pp. 14-23.

Musitu, G., y García, F. (2004): “Consecuencias de la socialización familiar en la cultura española”, Psicothema, vol.16, n. 2, pp. 288-293.

Oliveira, D. C. (2008): Análise de conteúdo temático-categorial: Uma proposta de sistematização. Revista de Enfermagem da UERJ, 16(4), 569-576.

Orozco Toro, J. A. (2010): “Comunicación estratégica para campañas de publicidad social”, Pensar la Publicidad, vol. 4, n. 2, pp. 169-190. 
Análisis de las campañas contra el castigo físico a menores en España...

Petty, R. E.; Briñol, P. y Priester, J. R. (2009): “Mass media attitude change: Implications of the Elaboration Likehood Model of Persuasion”, en Bryant, J. y Oliver, M. B. (eds.), Media effects: Advances in theory and research ( ${ }^{\text {rd }}$ ed). New York: Routledge, pp. 125-164.

Roberts, J. V. (2000): Changing public attitudes towards corporal Punishment: the effects of statutory reform In Sweden, Child Abuse y Neglect, vol. 24, n.8, pp. 1027-1035.

Rosser, A. (2015): Uso del castigo físico y otras estrategias de disciplina parental punitiva. I Congreso Internacional Psicologia Clinica En Niños Y Adolescentes. Madrid, 19-21 noviembre 2015.

Rucker, D. D.; Petty, R. E. y Briñol, P. (2014): “Social psychological foundations of social marketing”, en Steward, D. (Ed.), The handbook of persuasión and social Marketing (Vol 1): Santa Barbara, CA: Praeger Publisher, pp. 27-60.

Save the children (2003): Posicionamiento PPDI castigo físico y psicológico. La parentalidad positiva. Disponible en: http:// www.savethechildren.es/docs/Ficheros/250/posicionamiento_castigo.pdf. [Consultado el 16/09/16].

Save the children (2004): Castigo físico y psicológico en España. Incidencia, voces de los niños y niñas y situación legal. Madrid: Ministerio de Trabajo y asuntos sociales. Disponible en: http://www.savethechildren.es/docs/Ficheros/76/informeSC.pdf . [Consultado el 15/09/16].

Scott, J., Lewsey, J., Thompson, L y Wilson, P. (2013): Early parental physical punishment and emotional and behavioural outcomes in preschool children. Child: care, health and development. doi:10.1111/cch.12061.

Simons, D. A. y Wurtele, S. K. (2010): “Relationships between parents' use of corporal punishment and their children's endorsement of spanking and hitting other children”, Child Abuse and Neglect, vol. 34, n.9, pp. 639-646.

Smith, D. E.; Springer, C. M. y Barret, S. (2011): "Physical discipline and socioemotional adjustment among jamaican adolescents”, Journal of family violence, vol. 26, pp. 51-61.

Torío, S., Peña, J. V., e Inda, M. (2008): “Estilos de educación familiar”, Psicothema, vol. 20, n. 1, pp. 62-70.

Umeda, ,M., Kawakami, N., Kessler, R.C. y Miller, E. (2015): Childhood adversities and adult use of potentially injurious physical discipline in Japan. Journal of family and violence, 1(30), pp. 515-527.

Vázquez Sito, F. (1997). Análisis de contenido categorial: el análisis temático. Barcelona: Unitat de Psicologia Social -Universitat Autònoma de Barcelona.

Zolotor, A. J.; Theodore, A.D.; Chang, J. J. y Laskey, A. L. (2011): “Corporal Punishment and Physical Abuse: Population-based Trends for Three-to-11-year-old Children in the United States”, Child abuse review, vol. 20, pp. 7-66.

\section{Agradecimientos}

Este trabajo se ha realizado a partir de los resultados obtenidos en el proyecto europeo "PUNISH-ER. Eradication of the use of physical punishment on children within family and institutions” (JUST/2010/DAP3/AG/1337), financiado por la Iniciativa Daphne de la Unión Europea, en el que participaron Reino Unido, Italia, Alemania, Polonia, Estonia y España. 